\title{
PRÁCTICAS PEDAGÓGICAS EN LA FORMACIÓN DOCENTE: DESDE EL EJE DIDÁCTICO
}

\author{
María Ripoll-Rivaldo* \\ https://orcid.org/0000-0002-3248-7912
}

RECIBIDO: Noviembre 2020 / ACEPTADO: Febrero 2021 / PUBLICADO: Mayo 2021

Como citar: Ripoll-Rivaldo, María (2021). Prácticas pedagógicas en la formación docente: desde el eje didáctico. Telos: revista de Estudios Interdisciplinarios en Ciencias Sociales, 23 (2), Venezuela. (Pp.286-304).

DOI: www.doi.org/10.36390/telos232.06

\section{RESUMEN}

La formación inicial del docente es un proceso que tiene como propósito el desarrollo de competencias relacionadas con el saber didáctico para ejercer el ejercicio profesional, los sujetos en formación deben desarrollar una serie habilidades concretadas en propuestas didácticas, sobre todo, teniendo en cuenta las necesidades e intereses de los estudiantes, transformando los saberes teóricos en saberes prácticos. El objetivo del presente trabajo es formular lineamientos que fomenten la práctica pedagógica profesional en la formación docente para el ejercicio didáctico en el programa de Licenciatura en Pedagogía Infantil; basándose en los planteamientos de autores tales como: García García (2018), Feo Mora (2011), Ministerio de Educación Nacional (2016), entre otros. Desde la postura metodológica se asumió el enfoque cualitativo, apoyada por el paradigma interpretativo fenomenológico y para el análisis de los hallazgos se basó en la teoría fundamentada y su método comparativo continuo. Los resultados reflejan la necesidad de visualizar las prácticas pedagógicas profesionales desde el eje didáctico con el propósito de cualificar dicho proceso, como parte fundamental de la formación inicial del docente; ya que refleja la comprensión y aplicación de conocimientos prácticos y metodológicos. En cuanto a los supuestos teóricos deben ser componentes directos incluidos en el currículo de las instituciones de educación superior.

Palabras clave: Prácticas pedagógicas, formación inicial docente, eje Didáctico, pedagogía infantil, currículo.

\footnotetext{
* Licenciada en Ciencias de la Educación, Psicóloga, Magíster en educación y Doctorante en Ciencias de la Educación. Facultad Ciencias Humanas y Sociales. Programa de Licenciatura en Educación Infantil. Docente Líder de Investigación, Corporación Universitaria Minuto de Dios-UNIMINUTO. Colombia. Correo electrónico: mripollriva@uniminuto.edu.co
} 


\title{
Pedagogical practices in teacher training: from the didactic axis
}

\begin{abstract}
The initial teacher training is a process that aims to develop competencies related to didactic knowledge to exercise professional practice, the subjects in training must develop a series of skills specified in didactic proposals, above all, taking into account the needs and students' interests, transforming theoretical knowledge into practical knowledge. The objective of this work is to formulate guidelines that promote professional pedagogical practice in teacher training for didactic exercise in the Bachelor's Degree in Early Childhood Education; based on the approaches of authors such as: García García (2018), Feo Mora (2011), Ministry of National Education. (2016), among others. From the methodological stance, the qualitative approach was assumed, supported by the phenomenological interpretive paradigm and for the analysis of the findings it was based on the grounded theory and its continuous comparative method. The results reflect the need to visualize professional pedagogical practices from the didactic axis in order to qualify said process, as a fundamental part of the initial teacher training; as it reflects the understanding and application of practical and methodological knowledge. Regarding the theoretical assumptions, they must be direct components included in the curriculum of higher education institutions.
\end{abstract}

Keywords: Pedagogical practices, initial teacher training, Didactic axis, children's pedagogy, curriculum.

\section{Introducción}

La práctica pedagógica se constituye en un componente integral que impacta de manera notoria la formación inicial de todo docente, por lo anterior y con la finalidad de evaluar la situación actual de tan importante elemento, se plantea una investigación cualitativa con un enfoque interpretativo que facilitará el contraste de las posiciones que tienen los docentes frente su formación preliminar. Enmarcada en estas ideas, la práctica pedagógica profesional postulada por Trilla Bernet (2001), se puntualiza en la esencia de la formación docente para la práctica pedagógica, como protagonista de su misión dirigida a propiciar herramientas de descubrimiento en acción de gestión del conocimiento, representa el hacer desde el conocer.

En los últimos años se han realizado investigaciones de la UNESCO y en Centro Interuniversitario de Desarrollo [CINDA], que han arrojado informes acerca de la formación inicial del docente en el contexto internacional (Russell, et al. 2016), los cuales dan cuenta del desempeño de la labor docente y avances en la calidad de la educación universitaria; dentro de éstos últimos se encuentran nuevos modelos de formación, manuales de desempeño, desarrollo de competencias y sistemas de evaluación desde la perspectiva del estudiante en el campo de práctica y del apoyo y acompañamiento de los docentes tutores (Vaillant y Rodríguez, 2018).

De allí, que las políticas educativas y las acciones a nivel universitario, exigen que se analice y trate al problema de la práctica pedagógica desde la perspectiva de la investigación con una postura interpretativa desde el eje didáctico, ya que son muchas las investigaciones sobre la importancia de la integración proceso pedagógico y la práctica profesional (Díaz Velasco, 2016), (Espinosa, 2014), (Correa de Molina, et al., 2018), (Sacristán, et al, 2018). Sin 
embargo; hay muy pocas dirigidas desde la acción del ejercicio profesional didáctico en el proceso de enseñanza y aprendizaje, en consecuencia, que en esta investigación se busca cualificar la practica pedagógica profesoral a fin de construir supuestos teóricos que fomenten la práctica pedagógica profesional en la formación docente para el ejercicio didáctico en el programa de Licenciatura en Pedagogía Infantil.

Para Martínez et al, (2018). no solo las habilidades y competencias del docente deben ser objeto de cambio, sino también sus prácticas. Adicionalmente, García et al, (2018), sugieren que las instituciones educativas definen las herramientas y tácticas necesarias que le permitan ir a la par con los cambios generados, así poder implementar estrategias innovadoras para cumplir satisfactoriamente con las exigencias del usuario, ofreciéndole un servicio de alta calidad.

En virtud de lo expuesto, se evidencia que dentro del sistema de formación de educadores en Colombia, se establecen las disposiciones normativas alineadas con la política educativa nacional, estas se encuentran cimentadas en las necesidades de formación de los educadores a nivel local, regional y local (Nieva Chaves y Martínez Chacón, 2016). Por lo anterior, investigar sobre la práctica pedagógica profesional en las instancias preliminares, tiene una marcada relevancia en el contexto educativo nacional, ya que facilita la reflexión sobre la adquisición de los saberes, la apropiación de la investigación y la visibilidad de la relación teórico-práctica, logrando así un abordaje pertinente a la construcción del conocimiento pedagógico y las formas como el mismo puede ser promovido.

En este sentido, el ejercicio profesional desde la postura del eje didáctico cobra nuevas dimensiones, ya no se trata de impartir conocimientos y métodos de enseñanza, sino que se trata de una interacción socializada donde todos aprendan desde la postura del andamiaje presente y transformador. Por lo tanto, se trata de un cambio profundo, paradigmático pedagógico; de allí, la urgencia de una mayor apertura hacia los procesos de desempeño en transformación profesional en ejercicio didáctico, para mayor flexibilidad curricular y de pensamiento, que permita vincular efectivamente la profesionalización, la pedagógica, y el eje didáctico.

Por lo antes expuesto, es que resalta la importancia de la presente investigación que tiene por objetivo formular lineamientos que fomenten la práctica pedagógica profesional en la formación docente para el ejercicio didáctico en el programa de Licenciatura en Pedagogía Infantil; basados en los planteamientos de Feo Mora (2011), los cuales indican que la profesión docente requiere del dominio de una serie de elementos y procedimientos pertenecientes a la diversidad conformada por el contexto escolar, entre ellos se encuentra el eje didáctico, conformado por la planificación y la evaluación de los aprendizajes, así como también las estrategias de enseñanza (p.2) sin olvidar la integración escuela y comunidad, en función de las demandas de educandos, familia y comunidad.

\section{Práctica pedagógica: Un proceso integral enfocado en las necesidades del ámbito educativo.}

El sentido educativo de un docente, según Loaiza Zuluaga, et al., (2012), surge como consecuencia de sus experiencias anteriores, donde su práctica pedagógica debe ser entendida como un proceso de representación, formación y transformación, a los nuevos retos que la academia necesita; además partir de que su labor no solo implica transmitir 
conocimientos, su compromiso debe sustentarse con sentido de humanidad; con rigurosidad, calidad y exigencia.

La práctica pedagógica es considerada por Pineda-Rodríguez y Loaiza-Zuluaga (2018), una herramienta dinámica, cambiante y compleja que sirve como estrategia del saber, relacionada directamente con el entorno sociocultural donde se desempeña el docente, las relaciones con la práctica política, las teorías o disciplinas que la apoyan, entre otras. Específicamente, la práctica pedagógica contempla tres elementos metodológicos: la institución, el sujeto y el saber pedagógico.

En la actualidad, la formación docente se centra en una fundamentación epistemológica, teórica y práctica; estos elementos promueven una formación integral y de mejoramiento continuo, también posibilitan la acogida de competencias actitudinales y procedimentales, convirtiendo la práctica pedagógica en un proceso integral y que responde a las necesidades del ámbito educativo en los países (Hardman, et al, 2016).

Además, se debe tener en cuenta lo planteado por Maldonado, et al (2016), cada docente implementa diferentes enfoques y estrategias didácticas en su práctica pedagógica, donde, el factor motivación del docente que orienta su clase, se reflejará directamente en el desempeño académico del estudiante. Por lo cual, estás prácticas didácticas deben estar enfocadas en las necesidades de la colectividad estudiantil y empresarial. Pues como bien lo dice Páez (2006), pensar críticamente es una manera de vivir y de aprender que fortalece a la persona y, en el caso de los docentes, el pensar críticamente fortalece, consecuencialmente, a los estudiantes, a través de la práctica pedagógica

Por lo cual, en los tiempos actuales, se requiere de una práctica pedagógica en la que se produzca una enseñanza explícita, exposición frecuente a vocabulario dado y nuevo con atención a la profundidad, inmersión en un lenguaje rico, así como la necesidad de enseñar estrategias de enseñanza de palabras (Medina, et al, 2014).

En la presente investigación la concepción de práctica pedagógica profesional se asume como una representación social donde el eje didáctico, la comunicación, el currículo, los estudiantes y los saberes configuran un sistema para hacer de la educación un proceso integral, donde la didáctica facilitará la interrelación entre alumno-profesor, el desarrollo de habilidades sociales y resolución de problemas a través de sus capacidades de observar, descubrir, crear e innovar que desarrollen tanto alumnos como maestros en el proceso de enseñanza-aprendizaje.

\section{Formación inicial del docente: principios fundamentales enfocados hacia la interconexión entre la teoría y la práctica}

En la actualidad y como producto de la globalización y la mundialización se hace necesaria en Colombia una revisión profunda en cuanto de los planes y programas de formación inicial dirigido a estudiantes que hacen vida académica en las diferentes instituciones universitarias del país. En función de lo expresado, Imbernón (2001), establece cuatro principios fundamentales que respaldan la formación permanente del docente, que son "(a) Aprender continuamente de forma colaborativa (b) Conectar los conocimientos con nuevas informaciones (c) Reflexionar individual y colectivamente en la resolución de situaciones problemáticas de la práctica (d) Aprender en un ambiente formativo e interacción social" ( $p$. 30). 
En este contexto, Tejada Fernández (2009), agrega que el docente ha de planificar, impartir, tutorizar y evaluar acciones formativas, asumiendo así la formación docente como un conjunto de procedimientos teóricos, metodológicos, combinados con recursos didácticos, donde se adquieren herramientas, destrezas y las competencias necesarias para la administración de las unidades curriculares de un plan de estudio determinado; donde el trabajo diario de los mismos debe estar consustanciado con los fines filosóficos del sistema planteados por el Estado y con la implementación de herramientas que faciliten la interconexión entre la teoría y la práctica, para alcanzar la calidad de la formación y la actualización didáctica.

En la formación inicial del educador, es relevante valorar la práctica pedagógica profesional como un escenario de aprendizaje reflexivo, estructurado y enriquecedor desde el discurso pedagógico; asimismo, está encaminada a la formación de sujetos integrales, convirtiéndose de esta manera el docente en actores principales del proceso educativo (Owen, et al, 2018).

Por lo tanto, las competencias que pueda fortalecer y adquirir el futuro docente deben en todo momento tener como premisa generar el cambio y la innovación, toda vez que pueda dar respuesta o contrarrestar situaciones problemáticas en el seno de las institución educativas por intermedio de la revisión de su práctica pedagógica y en el compartir de ideas y experiencias con sus pares utilizando como referente la institución formadora y el contexto donde realiza las prácticas, de modo que se pueda evidenciar en el corto, mediano y largo plazo el efecto multiplicador tanto en lo individual como en lo colectivo.

\section{Eje Didáctico: herramienta para la planificación y evaluación curricular}

Las Prácticas Pedagógicas de los docentes, reflejan su impacto en el desempeño académico de los estudiantes; en los sistemas tradicionales de enseñanza donde el docente era el protagonista del proceso de enseñanza, el alumno debía demostrar lo que aprendió, o lo que el docente le enseñó, mediante el uso de su memoria, valorada a través de calificaciones establecidas en base a las evaluaciones realizadas, las cuales, miden su rendimiento académico; asimismo, el desempeño del estudiante además de depender de los resultados de las evaluaciones, también depende de sus participaciones y construcciones en el desarrollo de las unidades curriculares, las cuales son fomentadas por las estrategias didácticas del docente (Loaiza Zuluaga, et al. 2012).

De acuerdo a la naturaleza del presente artículo es necesario asumir el eje didáctico como la instrumentación que posee el docente como herramientas centradas en la planificación, la estrategia y la evaluación con miras a cumplir lo que establece el currículo. Desde esta perspectiva, el aprender a aprender está soportado teóricamente a partir de teorías del aprendizaje que configuran su aplicación teórica, es así que la educación como una práctica social, se apoya en dos elementos de transformación educativa, referidos a la socialización y la identidad.

Es por ello que las universidades deben resignificar el eje didáctico en su función formadora y transformadora, lo cual implica lograr en él un alto grado de reflexión y crítica hacia el aprendizaje. El docente en formación debe querer aprender de manera permanente. Deberá saber para qué aprende y cuál es la finalidad de dicho aprendizaje, su importancia y uso en su entorno social y cultural. 
Los autores W de Camilloni (2007), Medina y Salvador (2009), Segovia y Pérez Ferra (2016), García García (2018), Brailovsky (2020), coinciden en cuanto a la concepción y construcción del saber didáctico por parte del docente en formación, considerando que es un proceso que se alinea con la finalidad de diseñar y mediar en situaciones de aprendizaje, promoviendo la modificabilidad cognitiva, con el fin de contribuir en la planificación y organización de la acción pedagógica; dicho proceso se lleva a cabo en el quehacer cotidiano, en medio de una relación de co-aprendizaje entre colegas y estudiantes, utilizando medios y recursos simbólicos, escolares y reales.

Desde esta perspectiva Feo Mora (2011), considera a la "didáctica no como la práctica misma del enseñar" (p.67). De lo anterior se desprende la importancia dentro de la práctica pedagógica, ya que es necesario que dentro de la planificación, la estrategia y la evaluación se considere; ser participativo, flexible, la construcción permanente del proceso enseñanzaaprendizaje, la búsqueda de una propuesta integradora que pretende superar la tradicional factura entre las distintas áreas formativas, entre la teoría y la práctica, entre el pensamiento y la acción, entre los distintos actores y escenarios involucrados en dicho proceso.

En cuanto a la planificación dentro del eje didáctico, se asume como el proceso de elaboración sistemática de actividades dirigidas a los estudiantes con el objetivo de alcanzar los objetivos de un programa de curso mediante una serie de instrumentos y técnicas que señalaran el camino hacia la construcción del conocimiento (Carriazo Díaz, et al, 2020). Por lo tanto, la planificación educativa centra su atención en especificar los fines, metas y objetivos de un programa, este es un elemento importante de la práctica pedagógica ya que facilita el aprendizaje de los estudiantes para lograr esto el profesor pueda situarse en un realidad cultural, social y política.

De lo anterior resulta importante establecer que dentro del eje didáctico las estrategias están inmersas en el encuentro pedagógico y se realiza de manera presencial entre el profesor y el estudiante donde y a partir de ella se establece una comunicación real y pertinente que define las formas de adquisición de aprendizajes. Por último, la evaluación como un componente del eje didáctico y se concibe como los procedimientos consensuados en torno a los objetivos de un programa en función la valoración y descripción de los logros alcanzados por parte de los estudiantes en función de las metas de aprendizaje y enseñanza.

\section{Metodología}

El presente estudio, se desarrolló bajo un enfoque cualitativo, que según Strauss y Corbin (2016) "es una investigación que produce hallazgos a los que no se llega por medio de procedimientos estadísticos u otros medios de cuantificación" (p.56). Como supuestos cualitativos se siguen los mencionados por Creswell (2009), quien propone 6 pasos para sustentar dicho enfoque: (a) El investigador es el encargado de recolectar los datos. (b) Los datos obtenidos son descriptivos. Serán presentados en palabras, principalmente en palabras de los informantes; (c) El foco de la investigación está en la percepción y experiencias de los actores en las formas cómo ellos construyen el sentido de su experiencia; (d) Los esfuerzos se centran en el evento que está ocurriendo, así como en el resultado; (e) La atención se ubica en lo particular y los datos son interpretados en busca de lo singular de un caso, más que en la generalización y (f) El diseño es emergente, las interpretaciones son acordadas con las fuentes de datos, dado a que son sus realidades. 
En cuanto al tipo de investigación se concibe como interpretativa, apoyada en la teoría fundamentada, haciendo de esta una metodología única para este estudio por cuanto permite la creación de espacios para la acción de conglomerados profesorales que tengan como banderas de desarrollo la crítica y la autocrítica como camino hacia la comprensión de la naturaleza de los temas educativos en Colombia. Asimismo, se tiene en cuenta la utilización del método fenomenológico para la interpretación de los resultados, pues como lo define Martínez Miguéles (2009), este método se centra en el estudio de las realidades vivenciales tal y como se presentan y revelan a la propia conciencia con toda su concreción y particularidad.

En consecuencia, se adopta la Teoría Fundamentada y su Método de Comparación Continua (MCC), para llevar a cabo las categorías e igualmente para la fase de definición de categorías a través del programa Atlas TI. Abordar el estudio a partir de la metodología interpretativa-fenomenológica es hacer referencia a formas concretas de percibir y abordar la realidad, lo cual lleva a compartir posturas que coinciden en concebir dicha realidad como multi referencial, cambiante, cuyas explicaciones son un producto social y humano.

Por lo antes planteado, se hace necesario describir el procedimiento de acuerdo a los principios del método fenomenológico, que consisten en: (a) Primera Reducción: La liberación de los prejuicios; en esta etapa se pretendió la reducción fenomenológica a partir de aislar las teorías que en un determinado momento puede hacer que la subjetividad aparezca en las diferentes interpretaciones del sujeto. (b) Segunda Reducción: La descripción; en esta fase se realizó una revisión minuciosa de los elementos naturales y humanos que condicionan la realidad del sujeto. (c) Tercera Reducción: La comprensión de la esencia; esta fase comprende interpretar los rasgos distintivos de los informantes claves en procura de alcanzar delineamientos teóricos para optimizar las prácticaspedagógicas profesionales para el ejercicio didáctico de los estudiantes del programa de Licenciatura en Pedagogía Infantil de la Corporación Universitaria Minuto de Dios, sede Barranquilla.

El contexto de desarrollo de esta investigación fue los espacios académicos de Corporación Universitaria Minuto de Dios (UNIMINUTO) ubicada en Colombia, sede Barranquilla; por considerar que los docentes manejan información sobre la práctica pedagógica profesional de los estudiantes del programa de Licenciatura en Pedagogía Infantil. Como instrumento de recolección de información se realizaron entrevistas, dirigidas específicamente, a 120 docentes-tutores de las prácticas profesionales, quienes conformaron los sujetos informantes del presente estudio (ver tabla 1).

Asimismo, para la entrevista fue diseñado un cuestionario de treinta y cinco (35) preguntas que fue aplicado en los ambientes de aprendizajes de los informantes, basado en las categorías implícitas en las practicas pedagógicas tal como lo establece la literatura especializada que fue consultada para realizar el presente estudio; con la intención de extraer la información con más rigurosidad y las categorías para el desarrollo del proceso investigativo, relacionadas al rol del docente, componente disciplinar Vs. Componente práctico, pedagogía y didáctica, comunicación y evaluación. 
Tabla 1. Ubicación por semestre. Docentes-tutores del Programa de Lic. en Pedagogía Infantil

\begin{tabular}{ll}
\hline Semestre & Población \\
\hline $\mathrm{IV}$ & 18 \\
\hline $\mathrm{V}$ & 19 \\
\hline $\mathrm{VI}$ & 19 \\
\hline $\mathrm{VII}$ & 20 \\
\hline $\mathrm{VIII}$ & 16 \\
\hline $\mathrm{IX}$ & 14 \\
\hline $\mathrm{X}$ & 14 \\
\hline Total & $\mathbf{1 2 0}$ \\
\hline
\end{tabular}

Fuente: Elaboración propia

La información recolectada en el análisis de los hallazgos se expone de forma descriptiva e interpretativa, sobre la base del objetivo propuesto. La primera parte del análisis, busco: (a) interpretar las percepciones que manejan los docentes sobre la práctica pedagógica profesional y (b) las dificultades de los estudiantes en las prácticas pedagógicas del programa de Licenciatura en Pedagogía Infantil de la Corporación Universitaria Minuto de Dios, sede Barranquilla lo que permitió la configuración del análisis interpretativo que asignan los actores a los significados de las categorías de estudio.

\section{Resultados}

Dentro del contexto de la investigación, la primera reducción, la constituyó la codificación, (códigos y citas para el Atlas $\mathrm{Tl}$ ), establecidos como etiquetas o rótulos que se asignan a unidades de significado, con objeto de describir e inferir información acumulada, en vista de los resultados encontrados a través del programa informático, se logra representar gráficamente las relaciones entre las categorías, subcategorías y descriptores, en un sincretismo didáctico, es decir, codificados e interconectados entre sí en forma de redes estructurales o diagramas de flujo, que permitieron la teorización de la investigación.

\section{Categorías Abiertas}

Antes de realizar la mencionada codificación fue preciso realizar la categorización, donde se resaltan las palabras claves y relevantes de las entrevistas, así como de las observaciones suministradas por los informantes previamente seleccionados (Claret Véliz, 2012), lo cual permitió identificar las diferentes de las diferentes categorías y sub-categorías que fueron mencionadas de manera mayoritaria por los actores al momento de realizar las entrevistas, y que sirven como documentos primarios para aplicar el programa del Atlas Tl; entre las cuales resaltaron las siguientes:

(a) Las prácticas profesionales aparecen como un espacio académico.

(b) se pretende generar en el estudiante la capacidad de hacer propuestas de intervención pedagógicas,

(c) No tienen la misma relación de las planeaciones que les exigen en esta institución.

(d) En la práctica los estudiantes terminan realizando unas funciones diferentes a las estipuladas, 
(e) Práctica porque no cumplen con las funciones establecidas

(f) Eje didáctico en praxis pedagógica inexistente

(g) Participación Protagónica inadecuada,

(h) Epistemología en práctica pedagógica sin interacción protagónica.

Las afirmaciones obtenidas, en base a las entrevistas realizadas y a los referentes teóricos, permitieron elaborar la triangulación general enmarcándolas dentro de las categorías de estudio: (a) Práctica profesional, (b) eje didáctico en la transformación educativa.

\section{Categorías Axiales y selectivas}

El análisis de las entrevistas se inicia con los relatos de actores, donde a través de palabras que fueron categorizadas se expresan imágenes de un acontecimiento, una escena, experiencia, emoción o sensación, que para la presente investigación describen a las prácticas pedagógicas. Ahora bien, en relación a las categorías abiertas y axiales los hallazgos reflejaron las siguientes: (a) Clase planificadas de acuerdo a los programas, (b) Definición de Práctica Pedagógica(debilidades), (c) Deber ser Docente, (d) Actualización Eje Didáctico, (e) Habilidades Didácticas(Psicológicas y sociológicas que llevan a la obtención del conocimiento y (f) Debilidades y Acciones Docentes Erróneas, (g) Memorización, (h) La didáctica, (i) Evaluación, (j) Logro de conocimiento, (k) Comunicación efectiva,(I) Planificación, (m) Elementos que obstaculizan la practicas pedagógicas epistemología.

De la reducción fenomenológica se asumieron las categorías (selectivas): (1) eje didáctico, (2) programas de formación, (3) modelos pedagógicos, (4) Transformación de las practicas Pedagógicas y (5) Elementos que obstaculizan las practicas pedagógicas imprecisión en los conceptos de epistemología y teoría del conocimiento).

Es importante recordar que para las definiciones de dichas categorías, su codificación y estructuración se utilizó el programa Atlas TI, dado que trata los segmentos de texto marcados por los usuarios y/o codificados como objetos informáticos, donde sus resultados permiten nombrar las categorías, sub-categorías e indicadores resaltantes, se puede escribir información sobre los mismos y pueden ser incluidos en diagramas en forma de red para estructurar los resultados.

En otras palabras, a través del Atlas Ti se pudo establecer relaciones de diferentes tipos entre las citas, códigos y memos, esta relación lógica permite definir al analista los elementos importantes del análisis, los cuales vinculados entre sí pueden representarse gráficamente y editarse en las vistas de red. En consecuencia, se presentan los resultados a partir del método empleado, en las figuras 1,2 y 3 . 
Figura 1: Estructuras en Atlas TI de los informantes clave

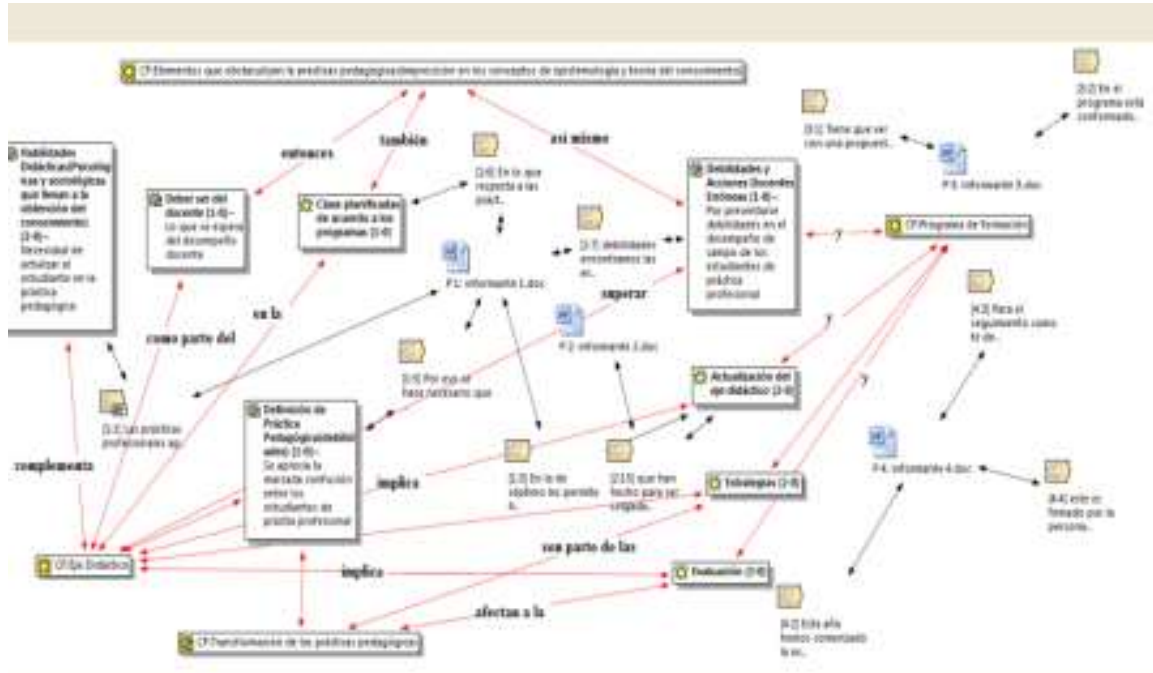

Fuente: Elaboración propia

Figura 2: Estructura particular de los informantes clave.

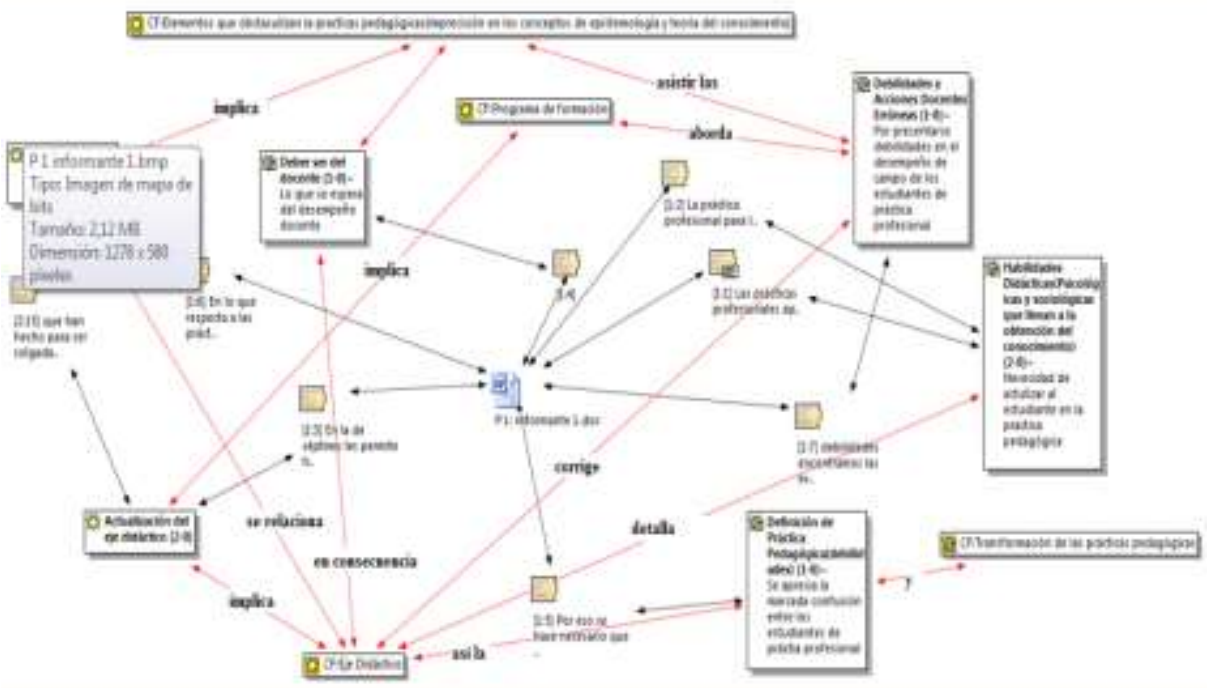

Fuente: Elaboración propia 
Figura 3.Integración de Todas las Estructuras

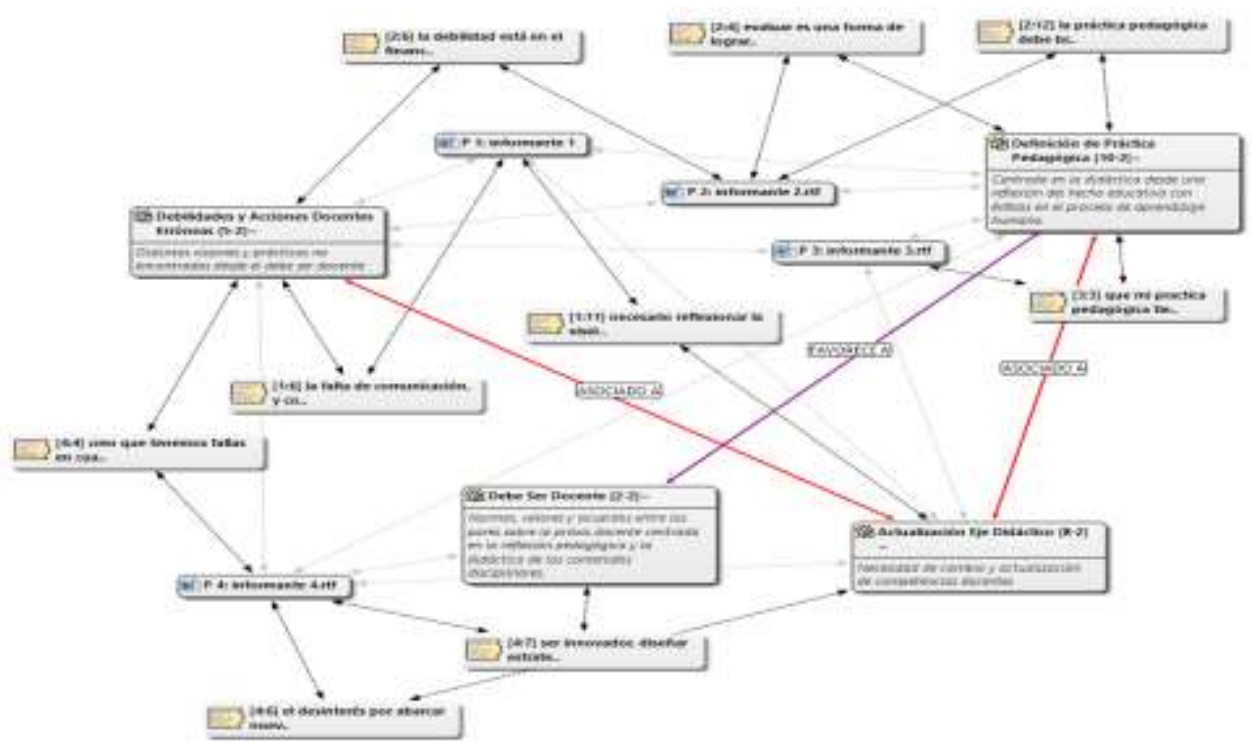

Fuente: Elaboración propia

La práctica pedagógica ha sufrido grandes transformaciones, es así como esta puede ser descrita como un proceso de autorreflexión, donde el docente en formación se apropia de los conocimientos necesarios para desarrollar las competencias que le permitan influir e impactar de forma positiva en los educandos y es por ello, que la práctica pedagógica ha recobrado un rol protagónico, ya que la misma debe reinventarse de forma constante teniendo en cuenta las nuevas tendencias educativas, para responder a los cambios que demanda el entorno social y económico, marcado por fenómenos como la globalización y las tecnologías de la información y la comunicación.

Desde la óptica de estas nuevas necesidades, la práctica del educador tiende a convertirse en un proceso esencial, donde las bases deben ser reestructuradas, dado que las condiciones del entorno actual distan de las tradicionales que se llevaron a cabo en los primeros sistemas educativos (Dole, et al. 2016), aunado a ello, la formación docente en el país debe exigir a las facultades de educación un objetivo inicial, como lo establece el artículo 9 de la Ley General de Educación: "formar un educador de la más alta calidad científica y ética, desarrollar la teoría y la práctica pedagógica como parte fundamental del saber del educador, fortalecer la investigación en el campo pedagógico y el saber específico" (Congreso de la República de Colombia, 1994).

Es necesario destacar que los futuros educandos o estudiantes de licenciatura en Colombia en primera medida deben de saber que la educación es un proceso humano ${ }^{1}$ y que

${ }^{1}$ Entender la educación como un proceso humano desde la perspectiva de los futuros educadores requiere tomar conciencia respecto
de la evaluación para el aprendizaje ya que, como lo resalta Alvares (2018, p. 105) este proceso implica enseñar es de doble vía, se 
su labor como educadores fundamentales en el desarrollo de los estudiantes, es por ello, que los futuros licenciados deben mirar que la práctica pedagógica se concibe como un proceso de auto reflexión, que se convierte en el espacio de conceptualización, investigación y experimentación didáctica. También es de mencionar que los futuros educadores, deben de potencializar y desarrollar competencias y actitudes que logren alcanzar el perfil de docente que integre los lineamientos de la educación, pero principalmente que vea en la teoría y en la práctica pedagógica como parte fundamental del saber del educador (Ministerio de Educación Nacional, 2016).

Por ende, estos aspectos de la formación del estudiante de licenciatura deben de ser direccionado por las estructuras curriculares ${ }^{2}$ que las universidades y las facultades de educación le tienen que brindar para que pueda desarrollar sus capacidades tanto el ámbito disciplinar como el ámbito pedagógico conlleva a que se tengan en cuenta que la práctica y acercamiento a los escenarios educativos les permitirán desarrollar los desempeños planteados por el Ministerio de Educación Nacional.

Específicamente, los desempeños antes mencionados se describen como: a) Acercarse, comprender, estudiar y proyectarse en el contexto de una institución educativa; b) Reconocer y comprender las dinámicas institucionales; c) Dimensionar su labor como generador de transformación social; d) Identificar problemas, necesidades contextualizadas y que sean posibles objetos de estudio; e) Establecer relaciones dialógicas con docentes de la institución educativa; f) Reconocer y comprender el cómo, porqué y para qué de lo que conlleva el proceso educativo; g) Reconocer, apropiar y usar los diferentes referentes de calidad, entre otros (Ministerio de Educación Nacional, 2016).

De esta manera, se puede determinar la relevancia de los escenarios donde se va desempeñar el futuro licenciado: ya que le permitirán ir construyéndose como formador y que a su vez las facultades educación deben ser los medios para que los educadores vayan integrándose a los diferentes contextos escolares que tienen matices y particularidades únicas que dependen de los actores, infraestructura, organización y entorno donde se desarrolle el plantel educativo.

Desde el Gobierno Nacional, las políticas de educación se imparten desde el Ministerio de Educación Nacional (MEN) y son proyectadas a un lapso de tiempo; para el caso colombiano desde la existencia de los planes decenales se ha buscado demarcar unas metas que se deben alcanzar en el intervalo de diez años; la Resolución 0241 guarda relación con el Plan Nacional de Desarrollo 2018 - 2022, planteado por el Presidente Iván Duque (2018),el cual tiene como objetivo brindar una educación de calidad y para lograrlo reconoce el papel de los docentes como los principales actores del proceso de formación, por lo tanto, se enfatizará en el fortalecimiento de las prácticas pedagógicas teniendo en cuenta la innovación y el desarrollo

aprende y enseña pero no siempre lo mismo, ni de la misma forma y mucho menos con la misma finalidad ya que los diversos grados de responsabilidad que se crean a raíz del vinculo educador-educando pues la evaluación es solo consecuencia del aprendizaje y por esta razón la evaluación formativa en futuros docentes cobra protagonismo al momento de medir los avances y logros alcanzados, es decir, "solo cuando aseguramos el aprendizaje podremos asegurar la evaluación, la buena evaluación que forma" (Álvarez, 2018, p. 105).

2Álvarez (2018, p. 108) la evaluación debe ser orientada por la enseñanza y el currículum. Es necesario innovar en la práctica docente para que los futuros educadores aprendan que "examen" no es sinónimo de "evaluación" pues la transformación curricular debe encaminarse a "lograr el aprendizaje significativo, entonces necesariamente se deben buscar nuevas ideas, formas nuevas, caminos de enseñanza distintos e innovadores" (Álvarez, 2018, p. 108) y ligar la concepción curricular con la práctica docente -especialmente en términos de evaluación- para alcanzar los fines propuestos. 
de las competencias profesionales, lo que implica necesariamente una mejora significativa del sistema de educativo, que permita garantizar un alto nivel de calidad de los egresados.

\section{Percepción de tutores sobre las prácticas pedagógicas}

En virtud de todo lo anteriormente planteado, se pudo apreciar que en UNIMINUTO, se percibe la práctica pedagógica profesional como una actividad pedagógica complementaria a la formación del futuro docente en el área disciplinar, y que adquiere su relevancia a partir de la relación permanente entre la universidad, la sociedad y el mundo laboral, tal como lo plantea Pineda-Rodríguez y Loaiza-Zuluaga (2018), al referirse a dicha práctica como una pieza fundamental para integrar los contenidos con el contexto en el que desarrollará su actividad académica.

También se percibe que en el proceso de formación, la investigación es uno de los eslabones fundamentales, dado que se convierte en el espacio pedagógico donde el estudiante reflexiona sobre su propia práctica, genera nuevos conocimientos y propuestas de transformación, coincidiendo esto con los planteamientos de Maldonado, et al (2016), al referirse al factor motivación del docente como determinante en el desempeño académico del estudiante.

Dentro de los lineamientos institucionales de UNIMINUTO, se pudo apreciar conforme a las afirmaciones de los entrevistados que se encuentran establecidos los propósitos, el rol de los tutores de práctica y los criterios de seguimiento y evaluación, lo cual demuestra el enfoque didáctico de los mismos, pues según los criterios de evaluación bajo el enfoque didáctico expuesto por Loaiza Zuluaga, et al (2012), se debe asumir el eje didáctico basado en las herramientas que utiliza el docente centradas en la planificación, la estrategia y la evaluación con miras a cumplir lo que establece el currículo.

\section{Dificultades de los estudiantes en las prácticas pedagógicas}

Todos los elementos que conforman el conjunto de oportunidades de mejora que tienen los estudiantes y señalados por los docentes tutores en el proceso de entrevistas, requiere que se tengan en cuenta algunas estrategias puntuales que contribuyan a mejorar los procesos de enseñanza. Específicamente, el futuro docente debe tener suficiente claridad sobre el contexto en el cual desarrollará su práctica, para así lograr identificar exitosamente las necesidades del aula intervenida; para lograr este propósito, la institución ofrece diversidad de recursos de apoyo (tutorías y orientaciones) para solventar sus dudas en el proceso, coincidiendo esto con los planteamientos expuestos por Hardman, et al (2016), quien habla de que la estrategia del saber, debe estar relacionada directamente con el entorno sociocultural donde se desempeña el docente.

En cuanto a las dificultades de los estudiantes en las prácticas pedagógicas, los informantes clave manifestaron las siguientes: (a)Dificultad en la Planificación general de la intervención, (b)Escasa descripción detallada del plan de clase, (c)Poca claridad en sus funciones, (d)Escasos conocimientos de lineamientos pedagógicos en la educación inicial, (e)Insuficiente relación teórico-práctica en la metodología implementada, (f) Falta de conocimiento de los lineamientos curriculares y $(\mathrm{g})$ dificultades en la comprensión de la epistemología de la didáctica. 
Estas dificultades se relacionan con los aspectos que señalan Imbernón (2001) y Tejada Fernández (2009), al indicar que en las prácticas pedagógicas basadas en el eje didáctico se tiene que tomar en cuenta los procedimientos teóricos y metodológicos, los recursos didácticos, el proceso de aprendizaje en un ambiente formativo e interacción social, las competencias necesarias para la administración de las unidades curriculares de un plan de estudio determinado, todo esto debidamente concatenado con los fines filosóficos del sistema educativo Estadal y con la implementación de herramientas que faciliten la interconexión entre la teoría y la práctica.

\section{Desarrollo de los postulados necesarios para el fortalecimiento del eje didáctico en la práctica pedagógica profesional}

Para esta sección se debe considerar que los postulados en la transformación del eje didáctico de la práctica pedagógica profesional se apegan al proceso emergente del cual emana los aspectos referenciales que aquí se desarrollan. Por lo que se ha de tomar en cuenta la capacitación del profesional docente, para la ejecución real de actividades, en un contexto de educación que trascienda en el marco curricular de formación de formadores desde el aprender a aprender, fundamentado en los argumentos de Feo Mora (2011), PinedaRodríguez y Loaiza-Zuluaga (2018) y Hardman, et al, 2016), tal como se muestra en el cuadro 1:

Cuadro 1. Postulados necesarios para el perfil de formación del estudiante basados en el eje didáctico en la práctica pedagógica

\begin{tabular}{|l|l|}
\hline Postulados presentes y necesarios \\
\hline Transformación & Eje didáctico \\
\hline Práctica pedagógica profesional & Ejercicio profesional \\
\hline Capacitación & Proyectos \\
\hline Conocimiento & Programas \\
\hline Habilidades & Calidad Pedagógica \\
\hline Dominio & Participación \\
\hline Experiencias & Comunidad \\
\hline Formación & Familia \\
\hline Competencias & \\
\hline
\end{tabular}

Fuente: Elaboración propia.

Los postulados mencionados en el cuadro 1, se pueden describir específicamente como:

- La capacitación para ejecución real de actividades, fundamentado en PinedaRodríguez y Loaiza-Zuluaga (2018), apremia a conocer y comprender la realidad como praxis, en el cual el profesor, debe permear las actividades de transformación desde las cualidades, potencialidades, eficacia y eficiencia de su práctica pedagógica enfocada en el aquí y el ahora, es decir, el tomar su realidad y no otra.

Conocimiento de problemas de transformación del eje didáctico así comprende la capacidad de reflexión permanente en la acción y sobre la acción, basado en Loaiza Zuluaga, et al (2012), para lograr la transformación creadora del acto educativo y de las condiciones que limitan el aprendizaje, al tiempo que se estimula el propio desarrollo profesional. 
- Habilidades para orientar la institución hacia su entorno, en base a lo planteado por Hardman, et al (2016), donde la misma concibe el estar preparado para comprender e interpretar los procesos de enseñanza aprendizaje más adecuados a la naturaleza de la situación educativa con apoyo de los elementos del entorno.

Habilidades para enseñanza adecuada tomando en consideración lo planteado por Segovia y Pérez Ferra (2016), García García (2018), en base a los proyectos en la búsqueda de la construcción y de la transformación de ese saber-hacer por uno emancipador, crítico, innovador, surgido de la investigación y de la toma de conciencia acerca del mejoramiento de su labor educativa y de su formación reflexivo y crítico.

Conocimiento de Estrategias que según Feo Mora (2011), permitan construir conocimientos donde la postura curricular profesional, ha de apegarse a la participación protagónica emancipadora en conducción de un proceso con preeminencia de aprenda de acuerdo a sus intereses individuales, por tanto, aproveche realmente el tiempo, siguiendo el propio esquema de pensamientos crítico emancipador.

En base a lo antes planteado, se asume que la práctica pedagógica profesional fundamentada en eje didáctico se sustentan en los postulados de acción, que se mencionan a continuación: (a) practica pedagógica participativa, práctica pedagógica de acción educativa, (b) reuniones y vinculación de formación constante, (c) corregir deficiencias en la práctica pedagógica, (d) vincular la practica pedagógica con aspectos técnicos la interacción pedagógica en logro de perfil de competencias asertivas, con un sentido que incluye la emancipación del aprendizaje y practica pedagógica socio-interactiva, (e) asociar la orientación con la norma institucional y (f) necesidad de orientación hacia el entorno.

De igual forma, capacitar a los estudiantes en formación con talleres garanticen: (a) la relación teórica y práctica, (b) orientar hacia la participación en los aspectos sociales, (c) la adecuación de los contenidos educativos a necesidades del entorno,(d) cumplir con necesidades del entorno desde el aprendizaje significativo, (e) el cumplimiento de funciones de exploración y orientaciones de desarrollo socio-afectivo y moral, (f) utilizar procedimientos de interacción que favorezcan la discusión, acción que le permita valorar cualitativamente y protagónicamente el proceso pedagógico educativo, (g) promover las reflexiones, aplicaciones y creatividad, y tomar en cuenta las experiencias y conocimientos previos, así como propiciar situaciones ideales para favorecer el aprendizaje activo, e incorporar las actividades didácticas de ideas previas, para desarrollar la creatividad y la construcción colectiva, de esta forma lograr desde el avance acelerado de la gestión pedagógica educacional y su introducción en la esfera critica, (h) el permitir visualizar la importancia del contexto para el análisis e interpretaciones, orientadas a comprender el sentido que tienen el tema analizado, que representa la descripción de un campo cubierto por la práctica y la teoría. 


\section{Fiaura 4: Teorización de la Realidad}

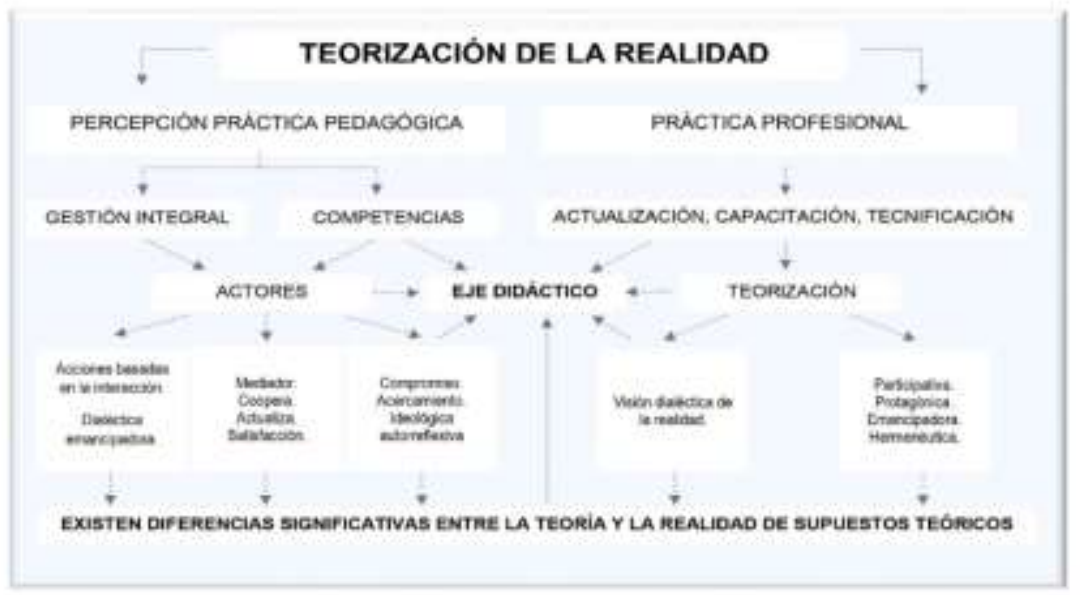

Fuente: Elaboración propia

La práctica pedagógica fundamentada en eje didáctico en ejercicio profesional, se muestra en la figura 4, la cual, debe ser discutida y aplicada, ya que permitirá que los involucrados alcancen cambios para la calidad educativa, con la integración escuela y comunidad, en función de las demandas de educandos, familia y comunidad. El estudiante en formación debe ser un moderador, gestor, coordinador, orientador, mediador.

Además, el estudiante tiene que ser un participante más de la transformación en el ejercicio didáctico profesoral y actuar como mediador que debe conocer: los intereses profesionales y sus diferencias individuales; a la vez, que proporcione una realimentación 0 refuerzo a cada una de las acciones pedagógicas, así ser, como lo mencionan Loaiza Zuluaga, et al (2012), el generador principal de la innovación, basado en la creatividad para el desarrollo, y proveer los recursos para optimizar la practica pedagógica; considerándola contextualizada, innovadora y creativa, y el ejercicio profesoral capaz, digno de respeto, consideración y confianza, mientras crea un ambiente armónico.

\section{Conclusiones}

Luego de analizados los resultados y culminado el proceso investigativo, se puede asumir, con relación al objetivo planteado en esta investigación que la práctica pedagógica profesional da primicia al descubrimiento, favorece la cooperación, colaboración y el intercambio de actividades y decisiones educativas que implican: (a) conocimientos, (b) capacidad para enfrentar y solucionar los problemas lo cual está asociado al proceso de ser sujeto como un ser social, lo que implica el promover la colaboración y el trabajo grupal ya que se establecen mejores relaciones con los demás, aprenden más, se sienten más motivados.

Asimismo, se asume que las dificultades que afrontan los estudiantes durante sus prácticas pedagógicas están en función de desarrollar nuevas estrategias didácticas que se podrían aplicar en las aulas por parte de los tutores de práctica, es por ello que se debe hacer 
mayor énfasis en el modelo praxeológico que se adelantan en los espacios académicos de Corporación Universitaria Minuto de Dios (UNIMINUTO) y así se convertirá en un mecanismo que permitirá solventar estas deficiencias, tanto a nivel universitario como profesional.

De este modo, impulsar acciones de mejora en la docencia, fortalecerá el rol del educador en sus dimensiones pedagógica, investigativa y evaluativa, por lo cual, se establecieron los postulados teóricos presentes y necesarios del perfil de formación del estudiante, además se plantearon mecanismos para llevar a cabo la práctica pedagógica fundamentada en eje didáctico en ejercicio profesional a fin de alcanzar mayores niveles de innovación y desarrollo para la institución y sus estudiantes adaptada a las exigencias curriculares en materia de educación.

\section{Referencias Bibliográficas}

Brailovsky, Daniel. (2020). Didáctica del nivel inicial en clave pedagógica (Primera edición). Editorial: Novedades Educativas. Argentina.

Carriazo Díaz, Cindy; Pérez Reyes, Maura y Gaviria Bustamante, Kathelyn. (2020). Planificación educativa como herramienta fundamental para una educación con calidad. Revista Utopía y Praxis Latinoamericana, 25 (3), Venezuela. (Pp. 87-95).

Claret Véliz, Arnoldo (2012). Proyectos comunitarios e investigación cualitativa. (Treceava edición). Editorial: Dirección de artes gráficas del M.P.P.D. Venezuela.

Congreso de la República de Colombia (1994). Ley 115 por la cual se expide la ley general de educación. Colombia.

Correa de Molina, Cecilia; Rodríguez Molina, Daniella; Gallego Quiceno, Danny y Simancas Trujillo, Ricardo. (2018). La armonía entre la teoría y la práctica: Formación docente. Ediciones Universidad Simon Bolívar. Colombia.

Creswell, John. (2009). Qualitative. Quantitative, and Mixed Methods Approaches (third edition). Editorial: SAGE. United States of America.

Díaz Velasco, Andrés. (2016). Editorial: La formación docente. Revista Lúdica Pedagógica, 2 (24), Colombia. (Pp. 05-08). https://doi.org/10.17227/01214128.4464

Dole, Sharon; Bloom, Lissa y Kowalske, Kristy. (2016). Transforming Pedagogy: Changing Perspectives from Teacher-Centered to Learner-Centered. The Interdisciplinary Journal of Problem-Based Learning, 10 (1), Cuba. (Pp.1-15). https://doi.org/10.7771/1541-5015.1538

Duque, Iván (2018). Plan Nacional de Desarrollo 2018-2022. Pacto por Colombia, pacto por la equidad. Extraído de: https://colaboracion.dnp.gov.co/CDT/Prensa/ResumenPND2018-2022-final.pdf

Espinosa, Julieta. (2014). La constitución de las prácticas de profesionalización de formación de docentes en México. Revista Perfiles Educativos, 36 (143), México. (Pp. 163179). https://doi.org/10.1016/S0185-2698(14)70615-4

Feo Mora, Ronald. (2011). Estrategias de aprendizaje que permiten aprender permanente. Revista Universitaria de investigación y Diálogo Académico, 7 (3), Venezuela. (Pp. 29-61).

García García, Vanessa. (2018). Desde una Didáctica Instrumental a una Didáctica Situada.

Revista de Estudios y Experiencias en Educación, 17 (34), Chile. (Pp. 129-138). https://doi.org/10.21703/rexe.20181734vgarcia1 
García-Guiliany, Jesús; Durán, Sonía; Parra Fernández, Margel y García Cali, Ernesto. (2018). Elementos y principios de la calidad de servicio en Instituciones de Educación Superior. En Prieto Pulido, Ronald y De la Hoz Reyes, Remberto. Marketing y Competitividad en las Organizaciones. Enfoques y Perspectivas. Ediciones Universidad Simón Bolívar. Colombia

Hardman, Frank; Stoff, Christian; Aung, Wan y Elliott, Louise. (2016). Developing pedagogical practices in Myanmar primary schools: possibilities and constraints. Asia Pacific Journal of Education, 36, United Kingdom. (Pp.98-118). https://doi.org/10.1080/02188791.2014.906387

Imbernón, Francisco. (2001). La profesión docente ante los desafíos del presente y del futuro. Extraído de: http://www.ub.edu/obipd/docs/la_profesion_docente_ante_los_desafios_del_presente $y$ del futuro imbernon f.pdf

Loaiza Zuluaga, Yasaldez; Rodríguez Rengifo, Juan; Vargas López, Hernán. (2012). La práctica pedagógica de los docentes universitarios en el área de la salud y su relación con el desempeño académico. Revista Latinoamericana de Estudios Educativos, 8 (1), Colombia (Pp. 95- 118).

Maldonado, Hugo; Vergel Ortega, Mawency; Gómez Vergel, Carlos. (2016). Prácticas pedagógicas e índices de creatividad en la enseñabilidad de la física electromagnética. Revista Logos, Ciencia \& Tecnología, 7 (2), Colombia. (Pp. 97104).

Martínez Miguéles, Miguel. (2009). Ciencia y arte en la metodología cualitativa. Editorial: Trillas. México.

Martínez, Olga; Steffens, Ernesto; Ojeda, Decired y Hernández, Hugo. (2018). Estrategias Pedagógicas Aplicadas a la Educación con Mediación Virtual para la Generación del Conocimiento Global. Revista Formación Universitaria, 11 (5), Chile. (Pp.11-18). http://dx.doi.org/10.4067/S0718-50062018000500011

Medina Rivilla, Antonio y Salvador Mata, Francisco. (2009). Didáctica General (Segunda edición). Editorial: Pearson educación. España.

Medina, Lorena; Valdivia, Andrea; San Martín, Ernesto (2014). Prácticas Pedagógicas Para la Enseñanza de la Lectura Inicial: Un Estudio en el Contexto de la Evaluación Docente Chilena. Revista Psykhe, 23 (2), Chile. (Pp. 1-13)

Ministerio de Educación Nacional. (2016). La práctica pedagógica como escenario de aprendizaje. Extraído de: https://www.mineducacion.gov.co/1759/articles357388 recurso 1 .pdf

Nieva Chaves, José y Martínez Chacón, Orietta. (2016). Una nueva mirada sobre la formación docente. Revista Universidad y Sociedad, 8(4), Cuba. (Pp.14-21).

Owen, Susanne; Palekahelu, Dharma; Sumakul, Toar; Sekiyono, Eko y White, Gerald. (2018). Systematic educational change and teacher skill-building in developed and developing countries: the importance of teacher peer learning groups. Teacher Development, 22 (4). United Kingdom. (Pp.447-463). https://doi.org/10.1080/13664530.2017.1403370 
Páez, Haydée. (2006). Planeamiento didáctico estratégico para el desarrollo del pensamiento crítico del estudiante. Una visión desde la práctica profesional docente. Revista Paradigma, 27 (1), Venezuela. (Pp. 349-363).

Pineda-Rodríguez, Yheny y Loaiza-Zuluaga, Yasaldez. (2018). Estado del arte de las prácticas pedagógicas de los maestros de las Escuelas Normales Superiores y las Facultades de Educación. Praxis, 14(2), Colombia. (Pp. 265-285). http://dx.doi.org/10.21676/23897856.2914

Russell, Tom; Fuentealba, Rodrigo y Hirmas, Carolina. (2016). Formadores de formadores, descubriendo la propia voz a través del SELF - STUDY. Editado por la Organización de estados iberoamericanos para la educación, la ciencia y la cultura (OEI). Chile.

Sacristán, José; Subirats, Mariana; Carbonell Sebarroja, Jaume; Rodríguez Martínez, Carmen; Álvarez Méndez, Juan y Adell, Jordi. (2018). Cambiar los contenidos, cambiar la educación. (Primera edición). Ediciones Morata, SL. España.

Segovia, Jesús y Pérez Ferra, Miguel. (2016). Aprendiendo a enseñar. Ediciones Pirámide. España

Strauss, Anselm y Corbin, Juliet. (2016). Bases de la investigación cualitativa: técnicas y procedimientos para desarrollar la teoría fundamentada. (Segunda edición). Editorial Universidad de Antioquia. Colombia.

Tejada Fernández, José (2009). Competencias docentes. Profesorado. Revista de Currículum y Formación de Profesorado, 13 (2), España. (Pp: 1-15) Extraído de http://www.redalyc.org/pdf/567/56711798015.pdf

Trilla Bernet, Jaume (2001). El legado pedagógico del siglo XX para la escuela del siglo XXI. (Primera edición). Editorial Graó, de IRIF, S.L. Barcelona, España.

Vaillant, Denise; Rodríguez Zidán, Eduardo (2018). Perspectivas de UNESCO y la OEI sobre la calidad de la educación. En Monarca, Héctor. Calidad de la Educación en Iberoamérica: Discursos, políticas y prácticas. Editor Dykinson, S.L. España.

W de Camilloni, Alicia. (2007). El saber didáctico (Primera edición). Editorial: Paidós. Argentina. 